\title{
Adaptive filter based on Monte Carlo method to improve the target tracking in radar systems
}

Khaireddine Zarai ( $\nabla$ khaireddine.zarai@fst.utm.tn )

Universite de Tunis El Manar

Cherif Adnane

Universite de Tunis El Manar

Research

Keywords: Radar, Monte Carlo, Extended KALMAN Filter, Tracking, PF, Random target

Posted Date: February 28th, 2020

DOI: https://doi.org/10.21203/rs.2.24844/v1

License: (9) This work is licensed under a Creative Commons Attribution 4.0 International License.

Read Full License 


\title{
Adaptive filter based on Monte Carlo method to improve the target tracking in radar systems
}

\author{
Zarai Khaireddine ${ }^{1}$, Cherif Adnane ${ }^{2}$ \\ ${ }^{1,2}$ Analysis and signal processing of electrical and energetic systems. Faculty of sciences of Tunis \\ .Tunisia
}

khaireddine.zarai@fst.utm.tn, adnane.cher@fst.rnu.tn, adnen2fr@yahoo.fr

\begin{abstract}
The state estimation and tracking of random target is an attractive research problem in radar system. The information received in the radar receiver was reflected by the target, that it is received with many white and Gaussian noise due to the characteristics of the transmission channel and the radar environment. After detection and location scenarios, the radar system must track the target in real time.

We aim to improve the state estimation process for too random target at the given instant in order to converge to the true target state and smooth their true path for a long time, it simplifies the process of real-time tracking.
\end{abstract}

In this framework, we propose a new approach based on the numerical methods presented by MONTE CARLO (MC) counterpart the method conventionally used named Extended KALMAN Filter (EKF), we showed that the first are more successful.

Keywords: Radar, Monte Carlo, Extended KALMAN Filter, Tracking, PF, Random target.

\section{Introduction :}

The nature of the signals reflected by any target, they are very noisy and interfered between them due to the nature of transmission channel and the nature of movement of this one. Most radar systems use a downstream processing chain where the first signal received at the baseband is processed., which serves to obtain the signatures of every target, then it is the phase of processing such as the positions, speeds and accelerations data into processor called tracker, which extracts the real direction and trajectory information's .System performance can be improved using adaptation between the information extracted from the sensor/processor and the design and transmission of subsequent illuminating waveforms.

To have a perfect tracking of the target, firstly, it is necessary to get a non-noisy signal which indicates the true target detection where the echoes are received in the baseband processing chain such as started by the Fast Fourier Transformation (FFT) block by the application of wavelet transformation method (WT) and discrete wavelet transform (DWT) method followed by a series of Doppler filtering. It called the phase of the mobile target identification (MTI), this later followed by the correlation phase of the real echoes, so that the function of the ambiguity determines the Doppler frequency, the position vector, the speed and the acceleration of every target. [2]

The Doppler Frequency and the distance Target-receiver is very important for knowing the direction of the target with regard to the radar. The estimation of the trajectory is made after three successive scanning in most by the antenna to command of phase, this number of noted scanning $\mathrm{M}=3$, is going to limit the false starts of targets. If vector positions and speeds being pre-calculated and were known, the radar software can smooth at present the data the most answered by movement, and draw the corresponding trajectory. 


\subsection{Related Works}

For target tracking and state estimation in radar system, various methods have been proposed in this framework, some involve special technologies like signal processing in real time, range rate measurement. To cope with these challenges, considerable researches have been undertaken in the literature. The majority of each approaches are concentred on target tracking scenario there is the processing of measurement-track in real time, using the recent analytic filter based on KALMAN Filter and Extended KALMAN filter.

We can quote a sequential unscented KALMAN filter (SUKF) proposed by [Zhansheng Duan, X.R. Li , Chongzhao Han , Hongyan Zhu ] [17] to solve the tracking problem with range rate measurement, also other approach based on Extended KALMAN Filter proposed by [Saab Bofors Dynamics, Jarfalla, Sweden ] [18] for tracking the position and the velocity of a manoeuvring target, based on non-linear model. PCRB and IMM for Target Tracking in the Presence of Specular Multipath are proposed by [Marcel Hernandez; Alfonso Farina] in 2019 ,a multi model has been developed based on the EKF to avoid the problem of tracking a non-cooperative target in the presence of specular multipath. [19]

In other hand, in order to avoid the problems issues of the degradation performances by the glint noise, [Jungen Zhang; Hongbing Ji; Qikun Xue] [20] they proposed an improved particular filter based on MARKOV Chain MONTE CARLO iterated Extended KALMAN filter ( MC MC -EKF) .

Unfortunately, the majority of these methods do not have effective solutions except that the latter have a relatively limited solution.

\subsection{PROBLEM STATEMENT}

The most tracking problems in radar systems are directly related by the Doppler filtering, the non-linearity, the size of the target, and the number of targets detected by every scanning. If the last one is important it pose a filtering problem affect to the tracking in real time. Besides the random (non-linearity) movement of every target it pose also a stochastic filtering problem according to SWERLING models [11], which causes the filter divergence phenomena, it is due to the combination of different kinds of non-linear measurements with different accuracies.

Trajectory estimation is posed as a continuous filtering problem with a simple closed -form solution when the divergence phenomena appear. The most successful tracking methods at present perform tracking by detection, the target is presented by an object model that can be detected in every independently scenario.

The advantage of using an object detector are that it naturally handles re-initialization if the target has been lost, and that it avoids excessive model drift. The detector yields the per-frame evidence for the presence or the absence of the target using the false alarm in discrete time.

In this paper, we are interest to improve the state estimation part to solve the tracking problems, new approach more used such as MONTE CARLO algorithm to avoid the divergence problem by EKF and make the tracking more efficient.

This paper is organized as follows. In section 2, we describe the Signal processing and tracking model in radar system. In section 3, presents the Adaptive algorithms for tracking the radar target. Simulation results are discussed in section 4 and section5, finally a conclusion and future works are given in section 6 . 


\section{Signal processing and tracking in radar system}

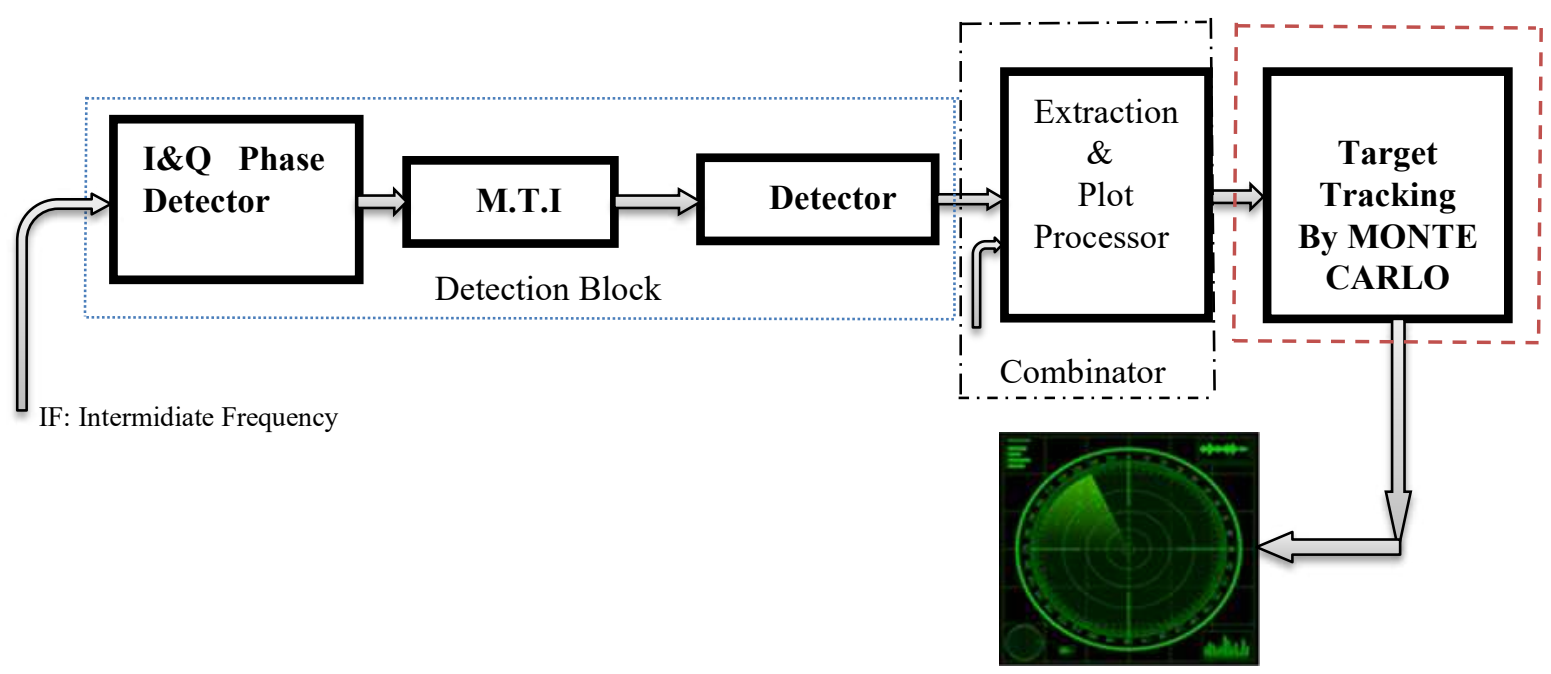

Figure 1. Block diagram

The signal processor is that part of the system which separates targets from clutter on the basis of Doppler content and amplitude characteristics. In modern radar sets the conversion of radar signals to digital form is typically accomplished after IF amplification and phase sensitive detection. At this stage they are referred to as video signals, and have a typical bandwidth in the range $250 \mathrm{KHz}$ to $5 \mathrm{MHz}$. The Sampling Theorem therefore indicates sampling rates between about $500 \mathrm{KHz}$ and $10 \mathrm{MHz}$. Such rates are well within the capabilities of modern analogue-to-digital converters (ADCs).

The signal processor includes the following components:

- The I\&Q Phase Detector,

- The Moving Target Indication (MTI)

- The Constant False Alarm Rate detection.

The complete proceeding may also be implemented as software in digital receivers.

The plot extraction and plot processing elements are the final stage in the primary radar sensor chain. The essential process is that of generating and processing plots as distinct from processing waveforms. The main components are:

- The plot extractor or hit processor (translates hits from the signal processor to plots),

- The plot processor (combines primary radar plots and minimises false plots)

- The plot combiner (combines primary and secondary plots, uses complementary features to minimise false alarms).

In this paper we are interest by the red block presented above in Fig. 1 which corresponds to the target tracking block.

\subsection{Data processing Algorithm in baseband}

Depending on the antennas rotation rate and pulse repetition frequency of a radar it will illuminate targets on a number of transmissions. In each range cell on each pulse period the signal processing decides whether a target is present, and if so produces a partial report, which defines the information available for this detection. The objective of the tracking process is to combine the data obtained from several reports per target into a single set of data for each target, in order to have reliable information about it. 


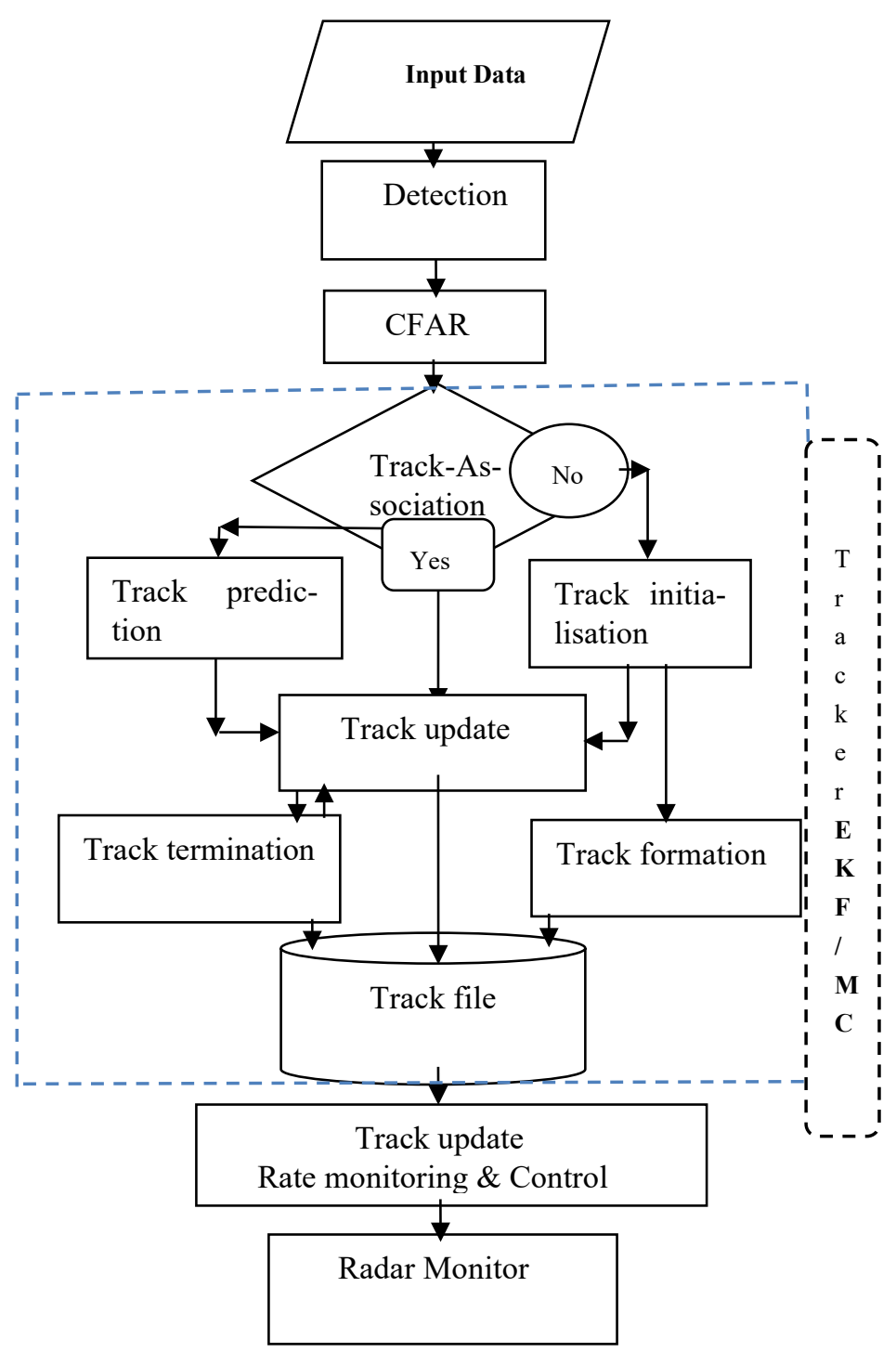

Figure2. Data processing Algorithm

\subsection{Target State estimation model}

According to the nature of movement of the target we can represent his model mathematical discreet in time. In the reality targets radars move with a time-varying speed so their accelerations varied abruptly, we say that they are (maneuvering) no linear targets, this characteristic raises a problem of the tracking.

We can describe the evolution of state of the target in the discreet time by the following state model:

$$
\text { (1) }\left\{\begin{array}{c}
X_{k}=F \cdot\left(X_{k-1}\right)+W_{k} \\
Y_{k}=H .\left(X_{k}\right)+V_{k}
\end{array}\right.
$$

Where $\mathrm{F}, \mathrm{W}, \mathrm{V}$ are respectively the system state function, the prediction noise and the measurement process noise.

The state model above determines the probability density $\mathrm{P}(\mathrm{k})$ for $\mathrm{X}(\mathrm{k})$ for $\mathrm{K} \geq 0$ and $\mathrm{Y}(\mathrm{k})$ for $\mathrm{K}>0$ and gives us at the beginning of the computation information about the initial state $X(0)$ of the target. The problem of computation in real time is apparent and for this reason we has applied a recursive algorithm using an optimal filter based on Hidden Markov Model (HMM) which checks the following two steps:

- The Prediction: a priori step

- The estimation: a posteriori step 


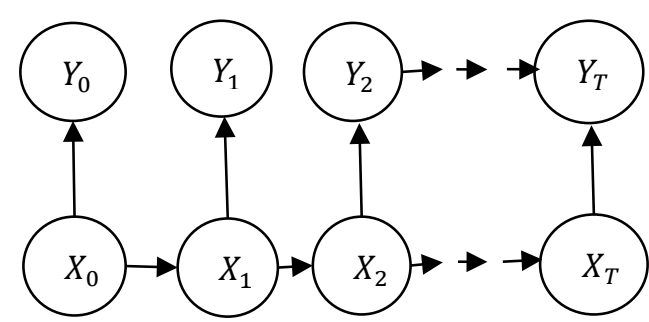

Figure3. Principle of optimal filter evolution according to HMM [1]

The approaches exposed by KALMAN such as the algorithm of extended KALMAN Filter (EKF) is going to return the equation of the observation $\mathrm{Y}(\mathrm{k})$ linearized with the equation of state $\mathrm{X}(\mathrm{k})$ in the following form:

$$
Y_{k}=\mathrm{H} . X_{k}, \text { Where } \mathrm{H} \text { it is the measurement function }
$$

In this context, the KALMAN filter (KF) was proposed by KALMAN and BUCY in 1964 as a solution to solve these problems when the target is linear, while Extended KALMAN filter (EKF) is supposed as partially solution for the non-linearity cases. The Both filters use various recursive algorithms, but they considered classic methods today, because the non-linearity of the target is considered among the target tracking challenges in radar systems. $[7][8]$

\section{Adaptive algorithms for target tracking in radar system}

In this section, we will present two different scenarios of target tracking methods in radar system using two different algorithms, such as Extended KALMAN filter counterpart the Particular Filer based on MONTE CARLO algorithm.

\subsection{The Extended KALMAN filter (Algorithm A)}

The EKF consists to derive the state and observation equations in the above state model (1), it uses the firstorder of Taylor series, and his algorithm is presented as follow: [1] [3] [5]. 
Initialization step

$$
\begin{gathered}
\hat{X}_{0} \leftarrow \bar{X}_{0} \\
P_{0} \leftarrow Q_{0}
\end{gathered}
$$

Loop: for

For $\mathrm{k}=1,2,3 \ldots$ do

Delay

$$
\begin{aligned}
& \hat{X}_{(k-1 / k-1)} \leftarrow \hat{X}_{(k / k)} \\
& P_{(k-1 / k-1)} \leftarrow P_{(k / k)}
\end{aligned}
$$

\section{Prediction step}

The Second order linearization around the predicted state: $F_{k} \leftarrow \nabla f_{k}\left(\hat{X}_{(k / k-1)}\right)$

Innovation step

$$
\begin{gathered}
X_{(k / k-1)} \leftarrow f_{k}\left(\hat{X}_{(k / k-1)}\right) \\
P_{(k / k-1)}=F_{k} P_{k-1} F_{k}^{T}+Q_{k}
\end{gathered}
$$

$$
\begin{gathered}
Y_{(k / k-1)} \leftarrow F_{k} \cdot \hat{X}_{(k / k-1)} \\
S_{k} \leftarrow H_{k} P_{(k / k)} H_{k}^{T}+R_{k}
\end{gathered}
$$

KALMAN Gain: The Signal noise ratio (SNR)

$$
K_{k}=P_{(k / k-1)} \cdot H_{k}^{T}+S_{k}
$$

\section{Correction and Estimation Step:}

Second order linearization around the estimate: $H_{(k / k)} \leftarrow \nabla h_{k}\left(\widehat{X}_{(k / k-1)}\right.$

$$
\begin{gathered}
\hat{X}_{(k / k)} \leftarrow \hat{X}_{(k / k)}+\left(Y_{k}-\hat{Y}_{(k / k-1)}\right) \\
P_{(k / k)} \leftarrow P_{(k / k-1)}-K \cdot H_{k} \cdot P_{(k / k-1)}
\end{gathered}
$$

Loop: end.

End.

\subsubsection{EKF limitation method}

The KALMAN Filter Process is the projection state estimation $\hat{X}_{k}$ on the orthogonal basic measurement space constructed by the innovation data $Y_{k}-Y_{(k / k-1)}$, it makes it possible to optimize the criterion of likelihood maximum, during the last years, the tracking problems in radar systems are improved relatively by KALMAN Filter and Extended KALMAN filter.

We note through the previous equations, that the covariance matrix sequence $P_{k / k}, P_{k / k-1}$ and the KALMAN gain $Y_{k}$ are an independent observations $Y_{k}$ so we can pre-calculate it, to reduce the calculation cost in real time.

The $f_{k}$ and the $h_{k}$ are non-linear functions, the optimal filter must linearize them around the predicted state $\hat{X}_{k-1}$ and around the current state $\hat{X}_{k}$ using the Taylor series to derive the state and the observation equations of the first and the second order.

The conventional problem, when the target is strongly non-linear, especially when the order of KALMANTarget Model is greater than 2, in this case the linearization of KALMAN becomes insufficient to make the system linear, therefore the occurrence of Filter divergence phenomena.

To avoid this problem we propose new approach as a Numerical Method based on Monte Carlo algorithm, to ensure the convergence of results in the worst cases. 


\subsection{The Numerical method: Monte Carlo method Particular filter (Algorithm B)}

The Monte Carlo method consists in representing the probability of the region observed by the concentration of their sequential samples $\mathrm{Np}$. It aim to get more distribution probability that it is generally impossible to calculate it analytically by EXTENDED KALMAN filter, through the following probabilistic interpolation:

$I(f)=E P[f(X) / Y]=\int_{1}^{N p} f(X) \cdot P(X / Y)$, Or the function of the empirical mean is defined as follows: $I_{N p}(f)=\frac{1}{N p} \cdot \sum_{i=1}^{N p} f\left(X^{i}\right)$, where $X^{i}$ represents the set of independent and identical random variables distributed by $1 \ldots \mathrm{Np}$. If the number of samples $N p$ tends to infinity, the filter converges to $\boldsymbol{E}(\boldsymbol{X})$ so we will have an error $\varepsilon=I_{N p}(f)-I(f) \approx 0$.

\section{General version of particulate filter based on Monte Carlo: S.I.R Algorithm}

\section{Initialization}

Loop: For $\mathrm{i}=1 \ldots \mathrm{Np}$ generate $X_{0}^{i} \sim p\left(X_{0}\right)$ with $W_{0}^{i}=\frac{1}{N p} ; N_{T h}=1 ; \varepsilon=0$

Loop: End

\section{Generation of particles related to their weights}

Loop: for $\mathrm{k}=1 \ldots \mathrm{K}$ to predict

- $\square$ Particle propagation over time

For $\mathrm{i}=1 \ldots \mathrm{Np}$ generate

$$
X_{K}^{i} \sim q\left(X_{k} / X_{0: K}^{i} ; Y_{k}\right) . . \text { End }
$$

Assign $T_{\text {samp }}=N_{p}$

Assign $Y_{1: K}$

Update of the importance weights and propagation over time:

For $\mathrm{i}=1 \ldots N_{P}$ calculate

End.

$$
\widetilde{W}_{k}^{i}=\widetilde{W}_{k-1}^{i} \cdot p\left(Y_{k} / X_{k-1}^{i}\right)
$$

Summing weights

$$
S=\sum_{i=1}^{N p} W_{k}^{i}
$$

Normalization of weights of importance

For $\mathrm{i}=1 \ldots N_{p}$ Normalize

$$
W_{k}^{i}=\frac{\widetilde{W}_{k}^{i}}{S}
$$

End

- Calculate the effective sample size: $\widetilde{T}_{e f f}=\frac{1}{\sum_{i=1}^{N p} W_{k}^{i^{2}}}$

- Calculation of Entropy: $\varepsilon=-\sum_{i=1}^{N p} W_{k}^{i} \cdot \log _{2} W_{k}^{i}$

- Calculation of optimal entropy: $\varepsilon_{\text {opt }}=\log _{2} N_{p}$

$$
\text { If } T_{\text {eff }}<T_{\text {samp }} \text { or } \varepsilon<\varepsilon_{\text {opt }} \text { do }
$$

\section{Call for systematic resampling}

Else $p\left(X_{0: k} / Y_{1: k}\right)=\sum_{i=1}^{N p} W_{k}^{i} \cdot \delta\left(X_{0: k}-X_{0: k}^{i}\right)$ End.

End. 
The Monte Carlo method uses an approach which generates independent samples according to the law $\mathrm{q}(\mathrm{X} / \mathrm{Y})$ (arbitrary samples law distribution) in order to obtain finally the desired posterior density $\mathrm{p}(\mathrm{X} / \mathrm{Y})$ : it is sequential importance sampling (SIS) followed by a sub-algorithm to avoid the divergence of the filter called: sequential importance resampling (SIR). [4][6][10][12][15] [16].

This method is better to solve the nonlinearity, non-Gaussian filtering problems and the divergence phenomena.

\subsection{Simulation model :}

In order to estimate the state $X$ of a random target, we must use a non-linear, non-stationary state space model, different scenarios has simulated using EKF and P.F approach during the time intervalt $t_{f}$, in this framework we present the following non stationary state space model:

$$
\left\{\begin{array}{c}
X(k+1)=0.5 * X+\left[25 * X(k) /\left[(1+X)^{2}\right]+8 \cos (1.2 k)+\right.\text { Process noise } \\
Y(k)=X(k)^{2} / 20+\quad \text { measurement noise }
\end{array}\right.
$$

\section{Simulation Results}

In this part, we use MATLAB software to simulate two different tracking scenarios, such as, presented in the theoretical part, it nevertheless, has a certain practical interest to show the contribution of the Monte Carlo methods compared by the classical methods such as the EKF which are sometimes successful and especially by proposing a simple solutions for a difficult tracking. We will compare the performance of EKF (Algorithm A) regarding the target state estimation problem. , after simulation we expect that the particular Filter based on Monte Carlo (algorithm B) considered as a solution for this problem, it's simple and efficient compared to the algorithm conventionally used.

\subsection{Simulation using Algorithm A}

Using the model $\left(^{*}\right)$ written in the previous part to simulate with EKF the state $\mathrm{X}$ of the target at the interval of time $\boldsymbol{t}_{f}$, in this framework, we initialize the parameters of simulation process in the following table:

Table1. Simulation parameters for Algorithm A

\begin{tabular}{clc}
\hline Parameters & \multicolumn{1}{c}{ Explanation } & Values \\
\hline $\mathbf{X}$ & The initial state of the target & 0.1 \\
\hline $\mathbf{Q}$ & The covariance matrix of process noise & 1 \\
\hline $\mathbf{R}$ & The covariance matrix of measurement noise & 1 \\
\hline $\boldsymbol{t}_{\boldsymbol{f}}$ & the simulation time interval in seconds & $50 \mathrm{~s}$ and $100 \mathrm{~s}$ \\
\hline $\mathbf{N S}$ & $\begin{array}{c}\text { The number of samples generated in } t_{f} \text { in first, the } \\
\text { second and the last times of simulation. }\end{array}$ & $500,2500,5000$ \\
\hline
\end{tabular}

\subsubsection{Parameters simulation Scenario A: we simulate with $\mathrm{N}=\mathbf{5 0 0}$ and $\mathrm{N}=\mathbf{2 5 0 0}$ samples in 50s}

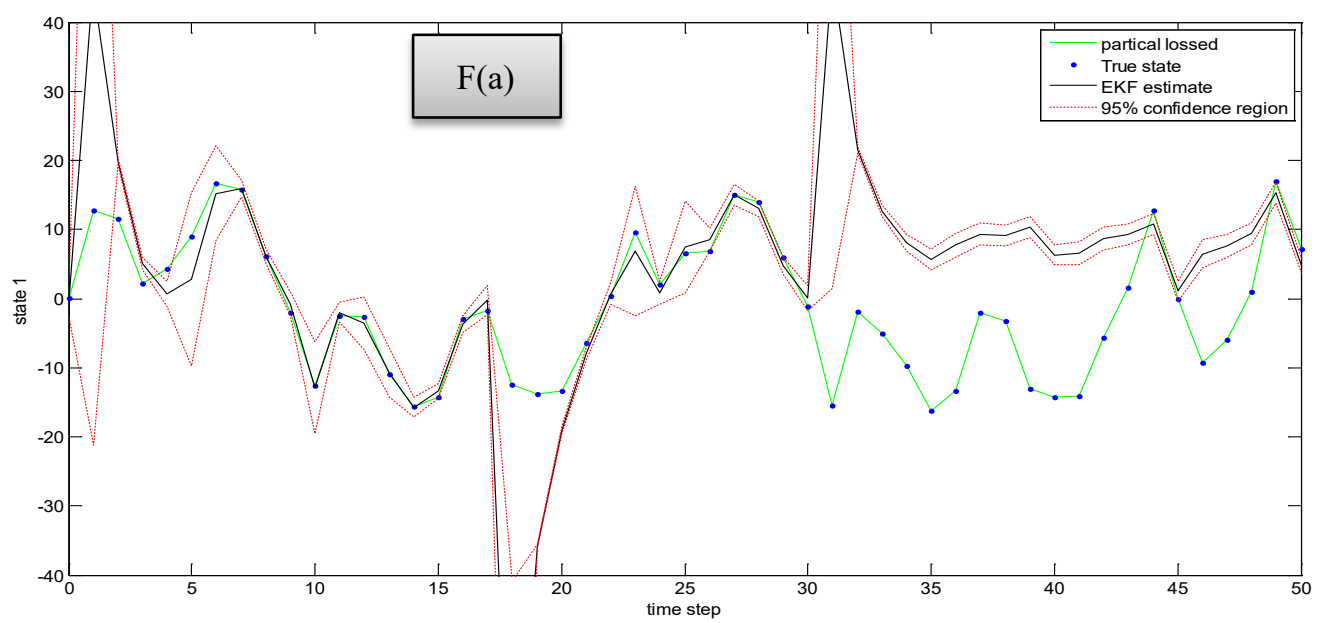

Figure 4.0 State estimated of the target (state $\left.\mathrm{N}^{\circ} 1\right)$ with $\mathrm{NS}=500$ and $\mathrm{T}=50 \mathrm{~s}$ respectively 


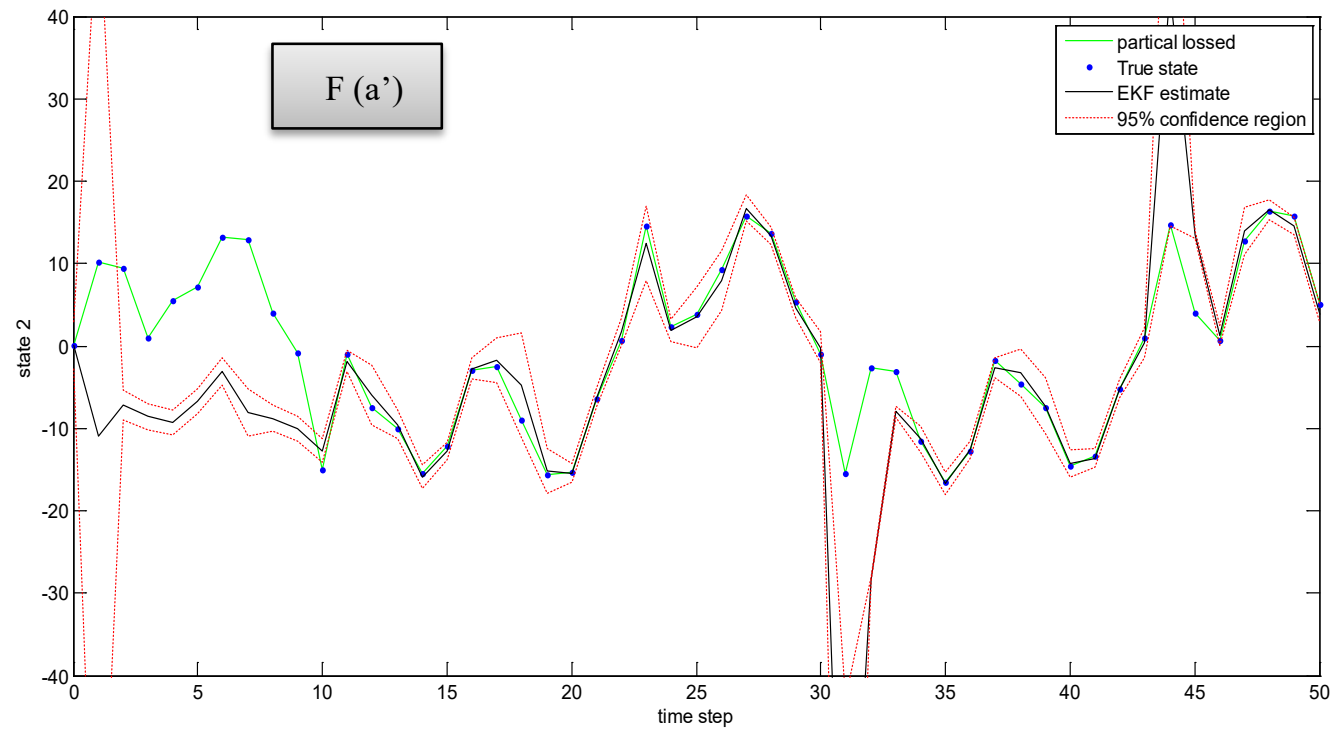

Figure 4.1 State estimated of the target $\left(\mathrm{F}\right.$ (a) et $\left.\mathrm{F}\left(\mathrm{a}^{\prime}\right)\right)$ with $\mathrm{NS}=\mathbf{2 5 0 0}$ and $\mathrm{T}=\mathbf{5 0}$ s respectively

4.1.2 Parameters simulation Step B: We Simulate with $\mathrm{NS}=\mathbf{5 0 0 0}$ samples in $\mathrm{T}=\mathbf{1 0 0 s}$.

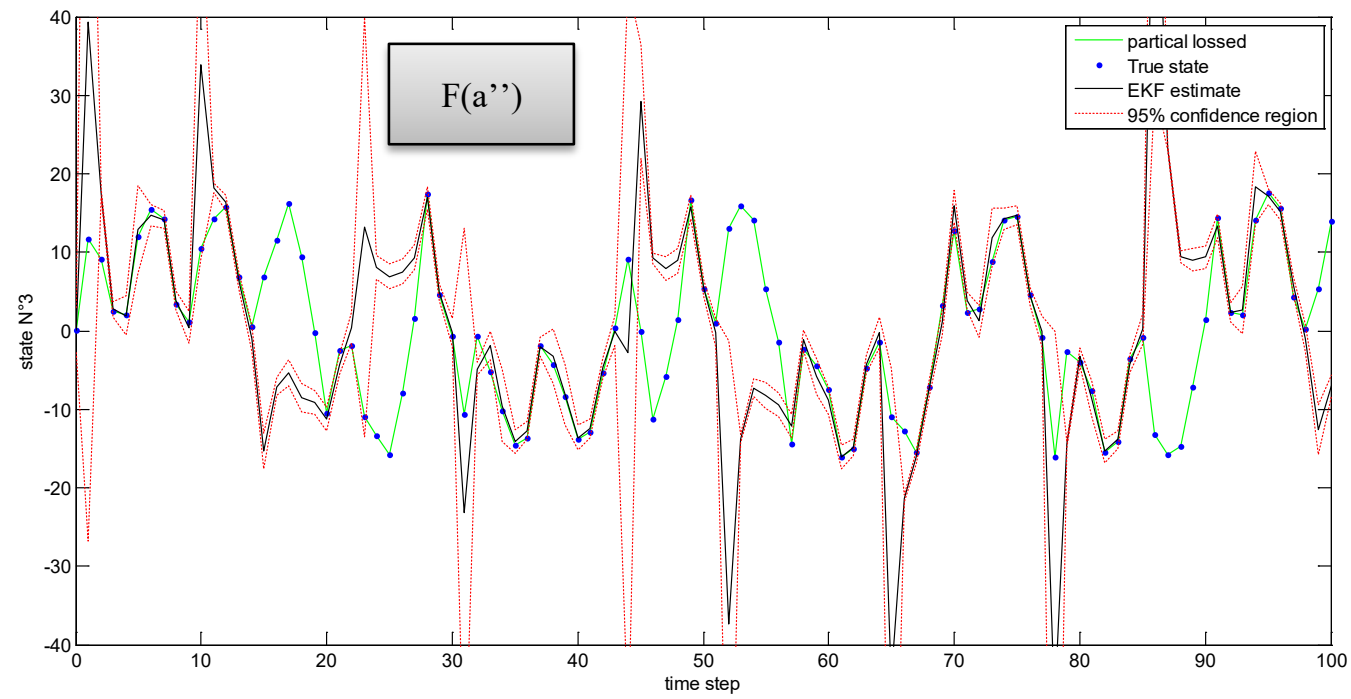

Figure 4.2 State $N^{\circ} 3$ estimated of the target with $\mathrm{NS}=5000$ and $\mathrm{T}=100 \mathrm{~s}$ 


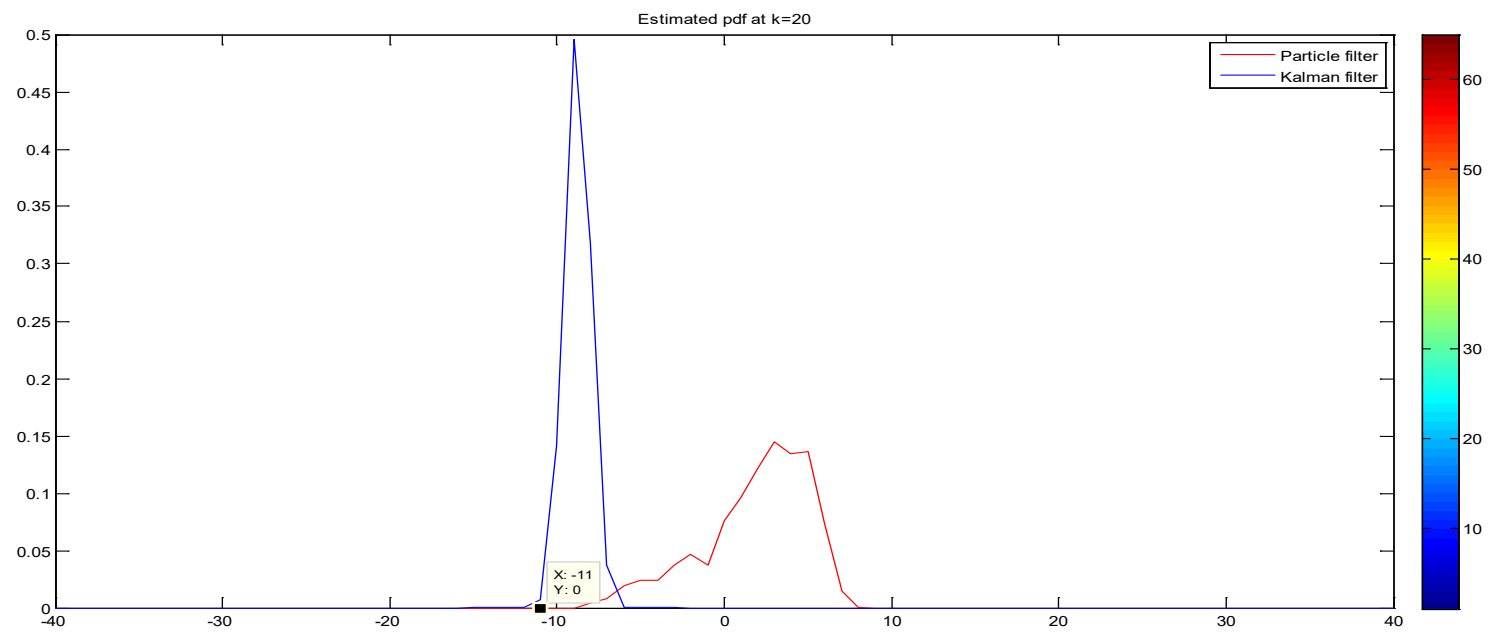

Figure 4.3 PF and EKF Estimation of Pdf using NS =500 and NS= 2500

\subsubsection{Interpretation}

Based on the obtained results in Figures F (a), F (a '), we note that the red curve which indicates the confidence parameter is identical to the black curve which indicates the response of the EKF estimator. we can say that the EKF gives a good state estimation of the target only when the interval of time is short with an average number of particles Ns= 2500 samples, otherwise it diverges (the red curve does not follow the response of the filter colored in black) as indicated by the curve F (a ") when the tracking period becomes greater than $50 \mathrm{~s}$ and the number of particles $\mathrm{Ns}=5000$.

In other hand, we can deduct from the figure 4.3 above that the extended KALMAN filter give 0.46 and 0.53 values of estimation Pdf (the probability density function), despite these values it gives us a low SNR not sufficient to the state estimation process in real time, especially when the number of samples $N \boldsymbol{s}$ is low. Contrary, The PF give us a $\mathrm{Pdf}=0.13$ value less than the EKF with an NSR considered as sufficient will be described in the next part.

\subsection{Monte Carlo Simulation for Performance Comparison : Algorithm B}

Using the same model above $(*)$, in this part we are interested to improve the target state estimation $\mathrm{X}$ during time interval [50 s and $100 \mathrm{~s}$ ], using Monte Carlo algorithm, in this context, we initialize the following simulation process parameters:

Table 2. Simulation parameters for Algorithm B

\begin{tabular}{clc}
\hline Parameters & \multicolumn{1}{c}{ Explanation } & \multicolumn{1}{c}{ Values } \\
\hline T (s) & number of time steps per second & $50 \mathrm{~s}$ and $100 \mathrm{~s}$ \\
\hline X0 & Initial state of the target & 0.1 \\
\hline R & Measurement noise (covariance matrix) & 1 \\
\hline Q & Process noise & 1 \\
\hline NS & Number of Monte Carlo samples per time step. & 2500 and 5000 \\
\hline
\end{tabular}




\subsubsection{Simulation Parameters Scenario A}

We Simulate with NS=2500 samples in $\mathrm{T}=50 \mathrm{~s}$.
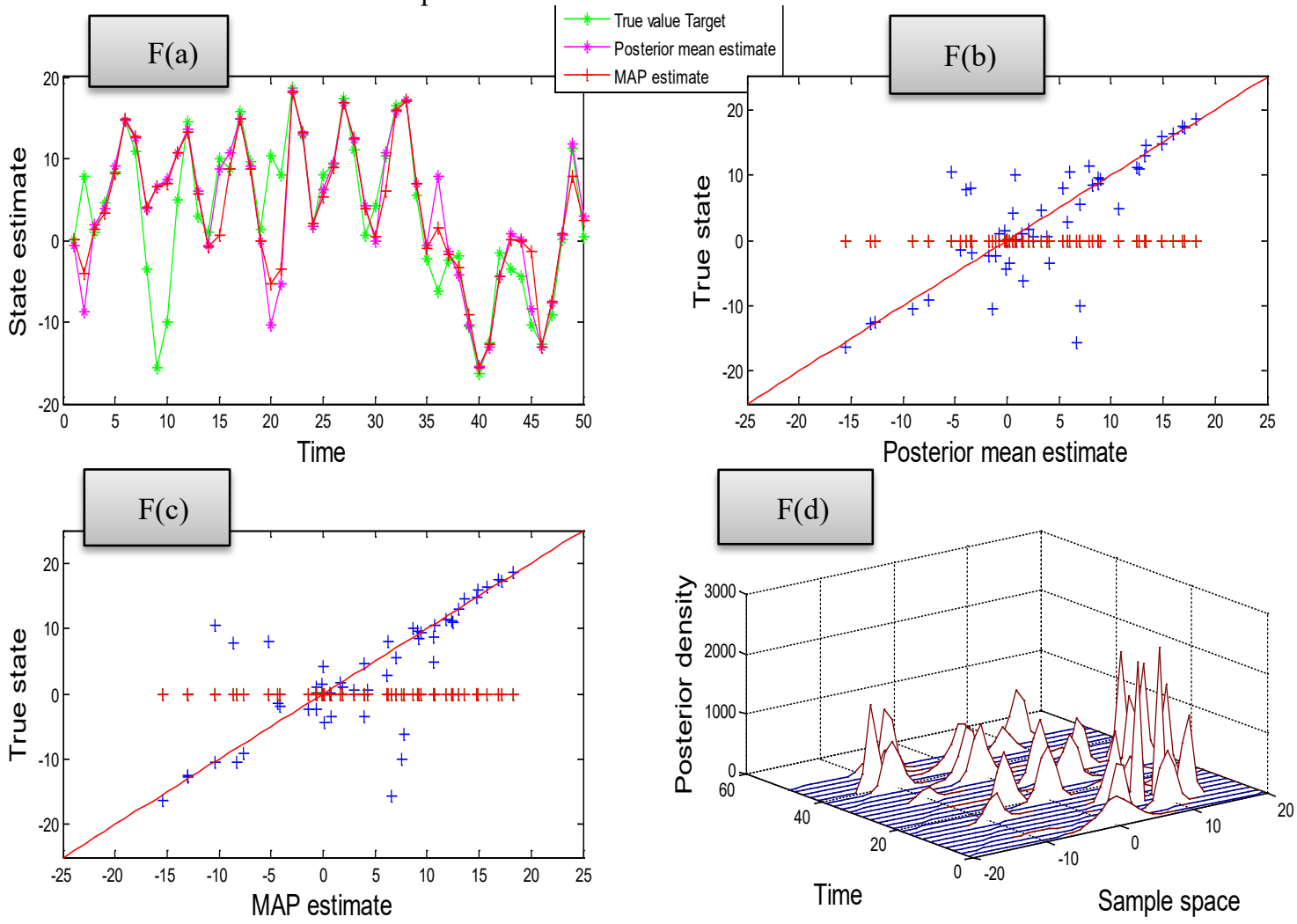

Figure 4.4 State estimation of manoeuvring target using SIR algorithm with $\mathrm{NS}=\mathbf{2 5 0 0}$ in $\mathrm{T}=\mathbf{5 0 s}$

4.2.2 Simulation Parameters: Scenario B

We simulate with: number of samples (NS) $=5000$ in $\mathrm{T}=100 \mathrm{~s}$ 

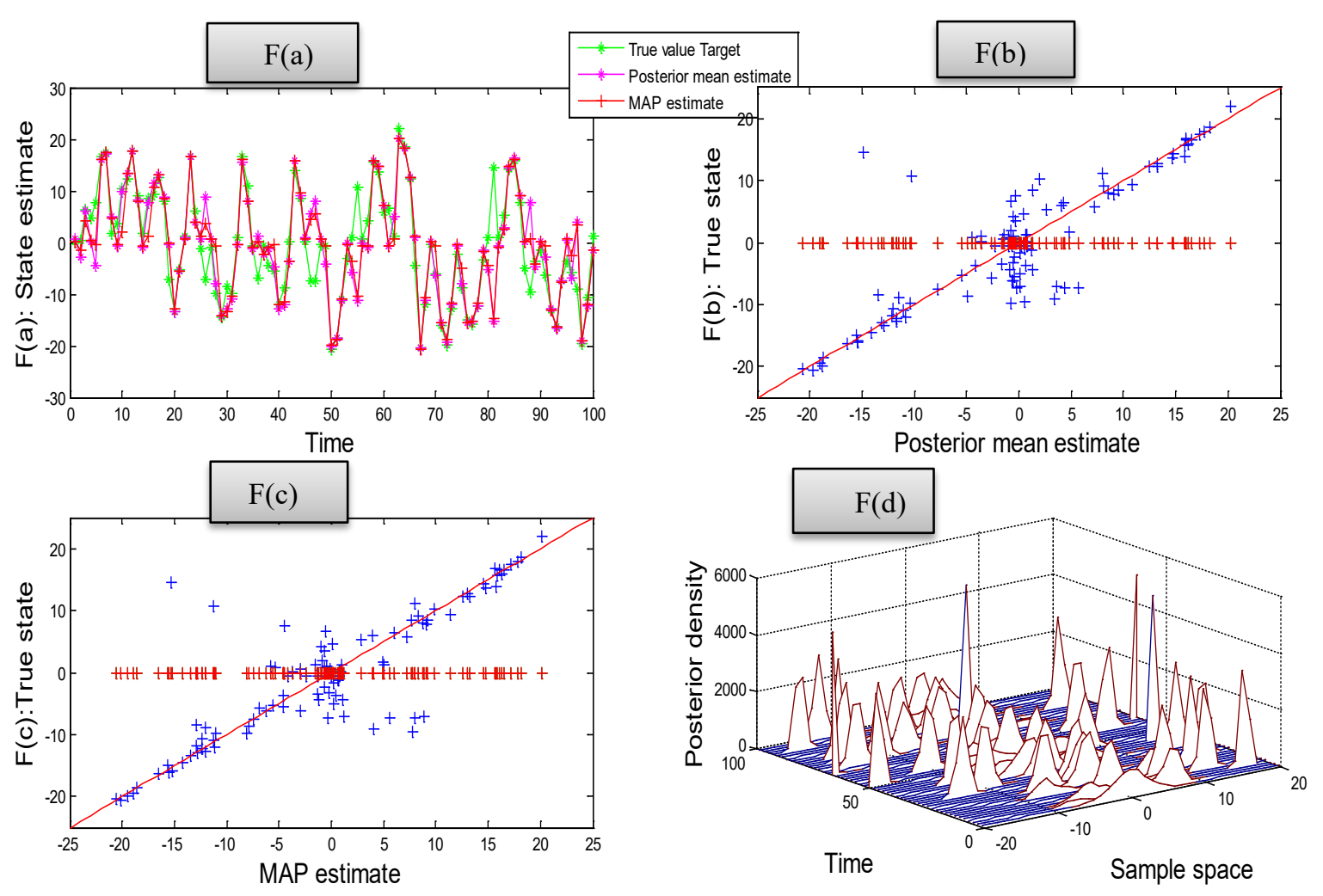

Figure 4.5 State estimation of a manoeuvring target using SIR algorithm with $\mathrm{NS}=\mathbf{5 0 0 0}$ in $\mathrm{T}=100 \mathrm{~s}$

\subsubsection{Interpretation}

F (a): the curve of the estimated state of the manoeuvring target, it calculates the error between the two curves of the true value of the target and the posterior mean estimate then gives us the MAP estimate (the estimated trajectory)

F (b): Present the true state in function of posterior mean estimate which must be linear between them.

F (c): Present the true state in function of the MAP estimate which must be linear between them to smooth the real trajectory.

F (d): The prior probability density function has the ambiguity function of the estimation process

According to figure 4.5: $\{\mathrm{F}(\mathrm{a})$ and $\mathrm{F}(\mathrm{d})\}$ we note that the probability of density function gives us more number of peaks in 100s and the sample space which is bound by the response of the particulate filter which gives us a more convergence with the target state estimation, especially when we increase the number of distributed particles.

\section{Comparative study}

\subsection{Obtained results}

The results obtained by scenario A and scenario B from MATLAB, are classified in the following table: 
Table 3. Comparative study table

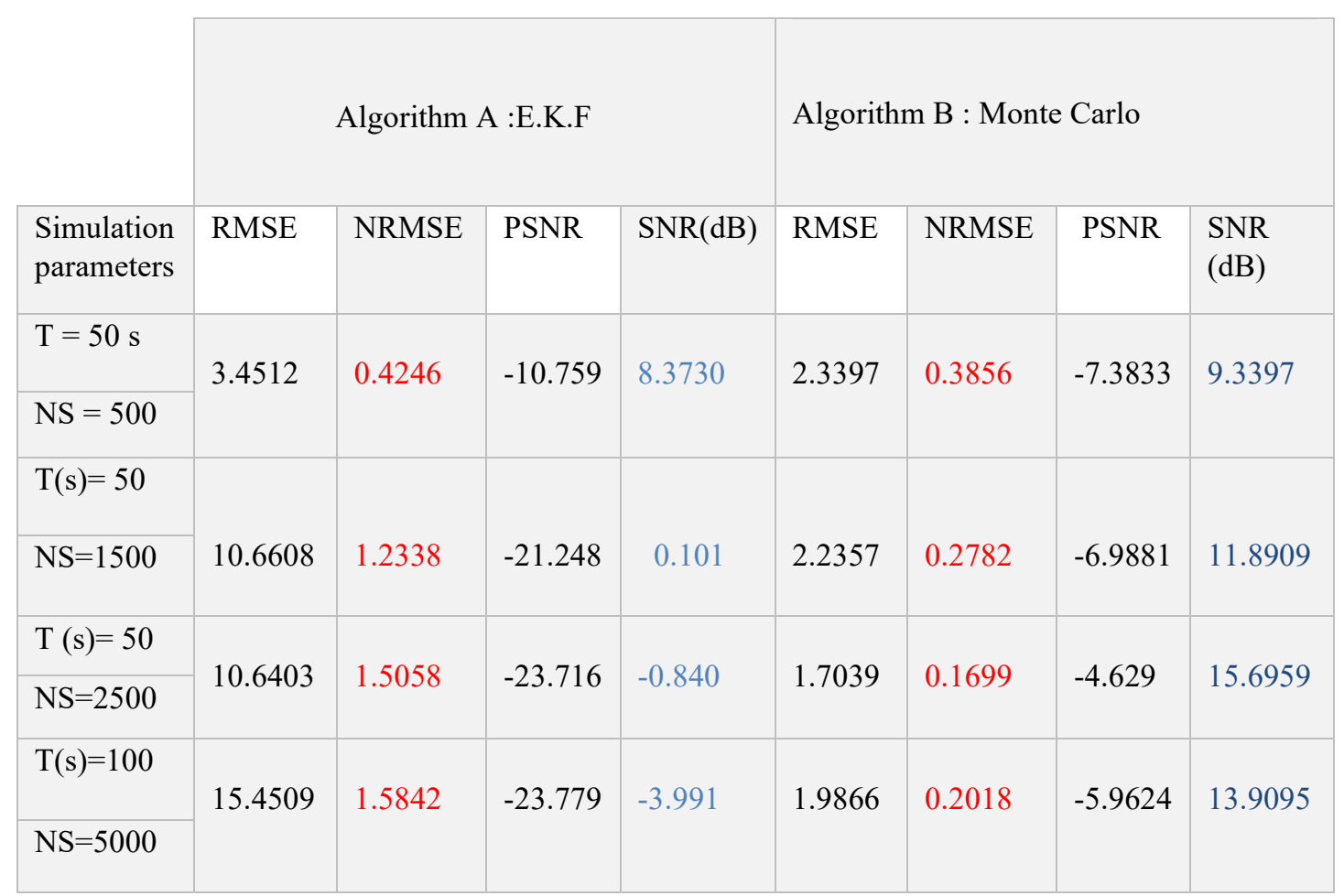

\subsection{Results and discussion}

The simulation results exhibits that the state estimations accuracy are not the same for all two filters. Yet, the PF shows better target state estimation with little errors.

Based on the results above in Table 3 , the theoretical results quoted in the second section are verified, the particular filter based on Monte Carlo methods is more suitable for estimating the state of a random target in the worst cases when the number of particles becomes high as NS $=2500$ and NS=5000 it gives an SNR $=15.6959$, SNR $=13.9095$ and low NRMSE between 0.1699 and 0.2018 it's sufficient to predict the true target state and smooths his real trajectory, Contrarily, the Extended KALMAN filter shows bad results and the error ratio (RMSE) it increases when $\mathrm{T}$ become $100 \mathrm{~s}$, he gives an NRMSE $=1.5842$ as long as we raise the number of particles $\mathrm{N}$. The KALMAN filter is diverged over the time, contrarily the PF shows faster convergence in short time and in long time $(\mathrm{T}=100 \mathrm{~s})$ than the other algorithm.

Therefore, it is possible to conclude that the Particular filter (algorithm B) estimates the state of the target more accurately than the EKF during 50 seconds and 100s using 2500 and 5000 number of Monte Carlo samples.

\section{Conclusion and future works}

In this paper, a novel method are proposed named the tracking by detection in order to improve the random target tracking in real time and to avoid the filter divergence phenomena , this one it called Monte Carlo algorithm.

The theoretical results are verified, we sought to overcome the constraints related to the nonlinearity of the target, and avoid the filter divergence phenomena, we got positive results from MATLAB simulations which confirms that the particular filtering (PF) based on Monte Carlo approach is more efficient in the complex situations that could not be empirically observed, compared to Extended KALMAN filter. The PF is fast in calculation, therefore it is capable to perform the real-time tracking in non-stationary white noise also avoid the divergence phenomena 
using the sub-algorithm called sequential importance resampling (SIR)., it can gives the necessary gain for the optimal filtering over a long period.

According to this approach, we improved the tracking of a random target in real time in a very noisy environment, which improves a significant part of the performance of the radar system, with this method, we aim in our future research to improve the multi-targets tracking in real time using a new data classification approach, associated with the Monte Carlo algorithm.

\section{Nomenclature}

EKF: Extended KALMAN Filter

MC: Monte Carlo

PF: Particular filter

$X_{k}$ : State estimation

$Y_{k}$ : Measurement function

$P_{k}$ : General Covariance Matrix

R: Covariance Matrix of measurement noise

Q: Covariance Matrix of Process noise

$K_{k}$ : KALMAN gain

$\widetilde{W}_{k}^{i}$ : Importance weight

$\varepsilon$ : Entropy function

$\mathrm{P}\left(X_{0: k} / Y_{1: k}\right)$ : the posterior Law

SIR: Sequential Importance Resampling

SIS: Sequential Importance Sampling

NS: number of Samples

Pdf: the probability density function

\section{DECLARATIONS}

1- CONSENT FOR PUBLICATION: In this paper just I used my own database obtained by radar observations and detections into research lab, to simulate the proposed scenario.

2- AVAILABILITY OF DATA AND MATERIAL: I can't share databases because I got it from a military security research lab, but using its data gives us positive results.

3- COMPETING INTERESTS : The authors declare that they have no competing interests

4- Funding: Because we are located in a lower-middle income country, Springer Journal community confirmed to me that the correction and the publication of this paper will be free.

5- AUTHORS 'CONTRIBUTIONS:

In this paper, we worked to improve the tracking of a random radar target, using a new approach based on particular filter called MONTE CARLO to solve the divergence problem appears over time by the recent filter used named extended KALMAN. The results obtained by MATLAB Software confirmed the theoretical results.

6- ACKNOWLEDGEMENTS : I would like to thank the thesis supervisor, Mr. Sharif Adnan, for all the support and assistance

7- AUTHORS' INFORMATION (OPTIONAL) : 
$8-$

- Zarai Khaireddine : PhD researcher at the Faculty of sciences of Tunis -Tunisia

- Pr.-Ing Cherif Adnane : Professor at the Faculty of science of Tunis- Tunisia

\section{References}

[1] Gennady Yu. Kulikov and Maria V. Kulikova. (FEBRUARY 15, 2016) the Accurate Continuous-Discrete Extended Kalman Filter for Radar Tracking, IEEE TRANSACTIONS ON SIGNAL PROCESSING, VOL. 64, NO 4.

[2] Dipl. ING. (FH) Christian Wolff, (2009), SM Sgt G.A.F. (Rtd.), Radar tutorial: Book 7 Radar Basics,radartutorial.eu.

[3] Florent Lafarge. (Edition 2009) "Introduction to KALMAN filtering Discrete KALMAN filter Theory and Applications".

[4] Jean-Charles NOYER. Gérard SALUT J. (1997) « Particle Filtering of RADAR Video Signals for Pointwise Targets ». Laboratory of Analysis and Architecture of CNRS Systems, Signal Processing - Volume $14-\mathrm{n}^{\circ} 1$.

[5] Yohan Petetin, Emmanuel Monfrini. (23-02-2012)."Kalman Filtering Applied to Mobile Target Tracking MAT450", François Desbouvrie, Paris.

[6] Khalil Jishy. (2011)"Tracking of moving targets in passive radar using Gaussian particle filtering". Doctoral thesis. National Institute of Telecommunications, Paris.

[7] C. Blanc; L. Trassoudaine ; J. Gallice,(5-28 July 2005),EKF and particle filter track-to-track fusion: a quantitative comparison from radar/lidar obstacle tracks, 7th International Conference on Information Fusion, IEEE.

[8] Hindawi Publishing Corporation. (Volume 2014).A Filtering Algorithm for Maneuvering Target TrackingBased on Smoothing Spline Fitting. Abstract and Applied Analysis .Article ID 127643, 6 pages

[9] Florent Lafarge. (2009). Introduction to Kalman filtering Discrete Kalman fi lter theory\& applications.book.Paris.

[10] Carine Hue. Jean-Pierre Le Cadre and Patrick Pérez. (FEBRUARY 2002) Sequential Monte Carlo Methods for Multiple Target Tracking and Data Fusion. IEEE TRANSACTIONS ON SIGNAL PROCESSING, VOL. 50, NO. 2.

[11] Kristine L. Bel, Christopher J. Baker, Graeme E. Smith. (Issue: 8, Dec. 2015) Cognitive Radar Framework for Target Detection and Tracking, IEEE Journal of Selected Topics in Signal Processing, Volume: 9.

[12] Min-Hyun Cho1, Min-Jea Tahk. (July 3-6, 2017). Modified Gain Pseudo-Measurement Filter Design for Radar Target Tracking with Range Rate Measurement, 25th Mediterranean Conference. Valletta, Malta.

[13] Christian Wolf, (20.12.2009), local tracking Book 1, Radar Basics "Mathematically Basics of Radar Technology", (18 pages), radartutorial.eu.

[14] BrankoRistic .M,SanjeevArulampalam. (June 2003).Tracking a manoeuvring target using angle-only measurements: algorithms and performance, Volume 83, Issue 6, Pages 1223-1238.

[15] Anton Milan, Konrad Schindler, and Stefan Roth.(OCTOBER 2016).Multi-Target Tracking by Discrete-ContinuousEnergy Minimization,IEEE TRANSACTIONS ON PATTERN ANALYSIS AND MACHINE INTELLIGENCE, VOL. 38, NO. 10. 
[16] Rickard Karlsson, Fredrik Gustafsson. (2017). Monte Carlo data association for multiple target tracking, IEEE.

[17] Zhansheng Duan, X.R. Li , (2005), Chongzhao Han, Hongyan Zhu : Sequential unscented Kalman filter for radar target tracking with range rate measurements, IEEE.

[18] Saab Bofors Dynamics, Jarfalla, Sweden, (May 17, 2015), a constrained extended Kalman filter for target tracking, Volume: 11 issue: 5. IEEE.

[19] Marcel Hernandez; Alfonso Farina, (2019), PCRB and IMM for Target Tracking in the Presence of Specular Multipath, IEEE Transactions on Aerospace and Electronic Systems.

[20] Jungen Zhang ; Hongbing Ji ; Qikun Xue ,( 2009 ), A new approach based on particle filter for target tracking with glint noise, IEEE International Conference on Systems. 
Figures

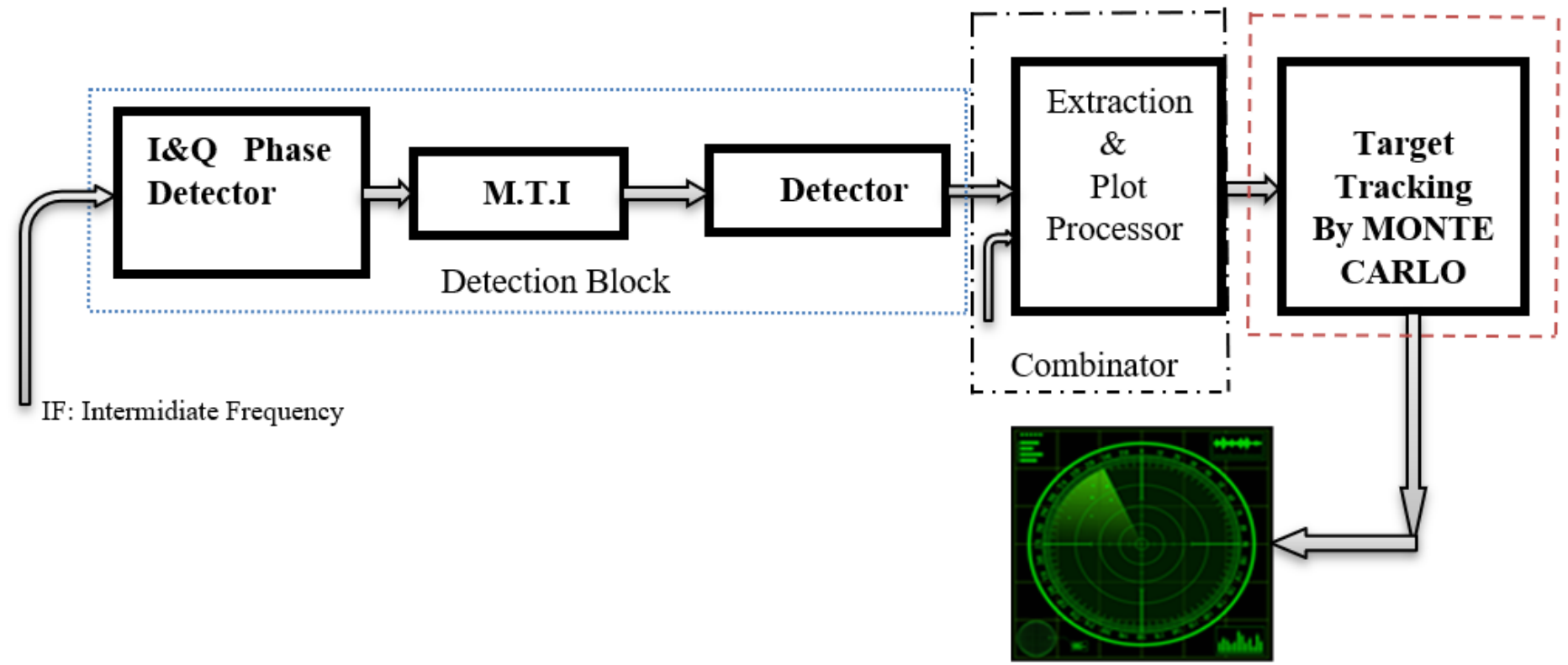

Figure 1

Block diagram 


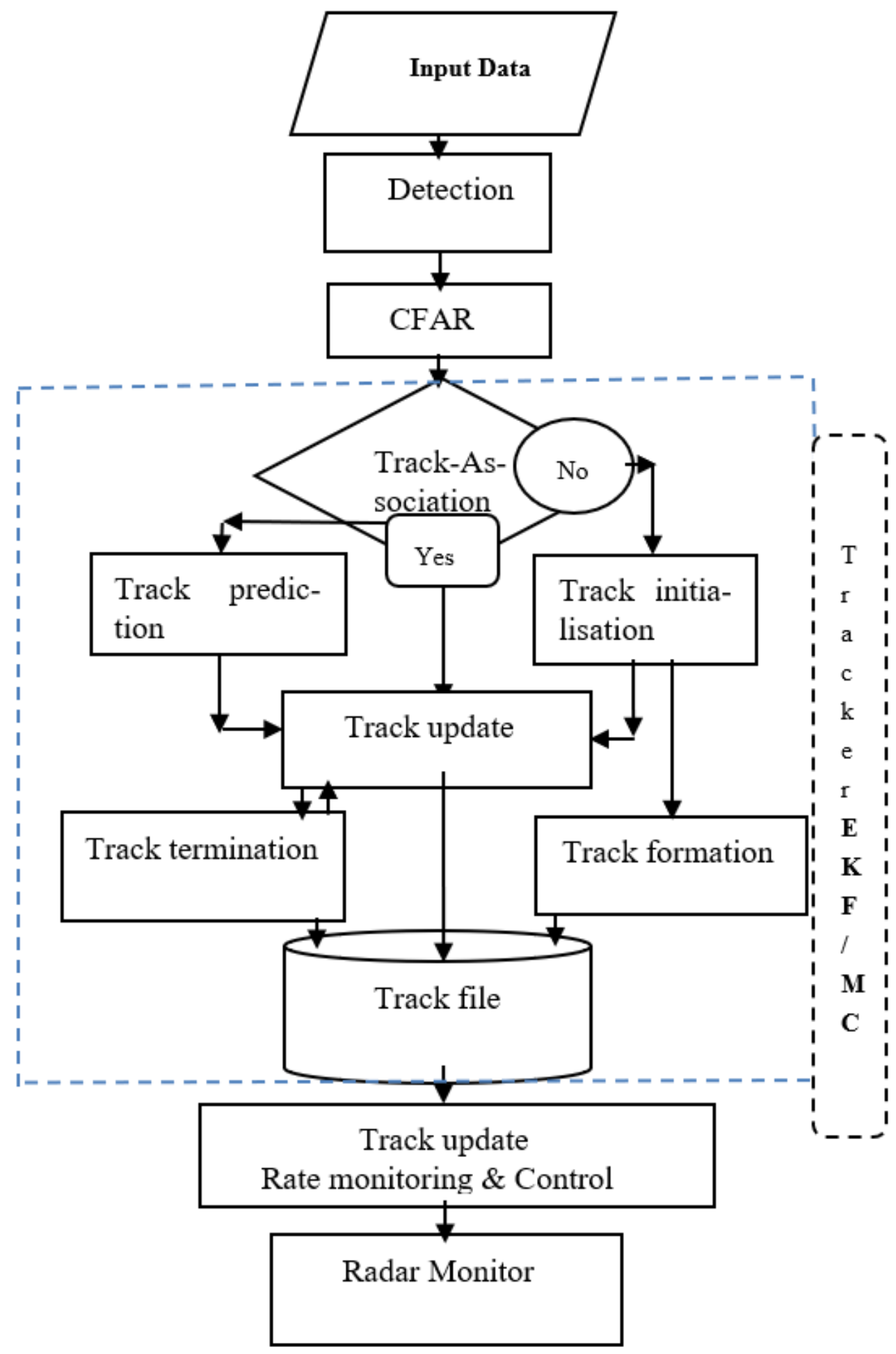

Figure 2

Data processing Algorithm 


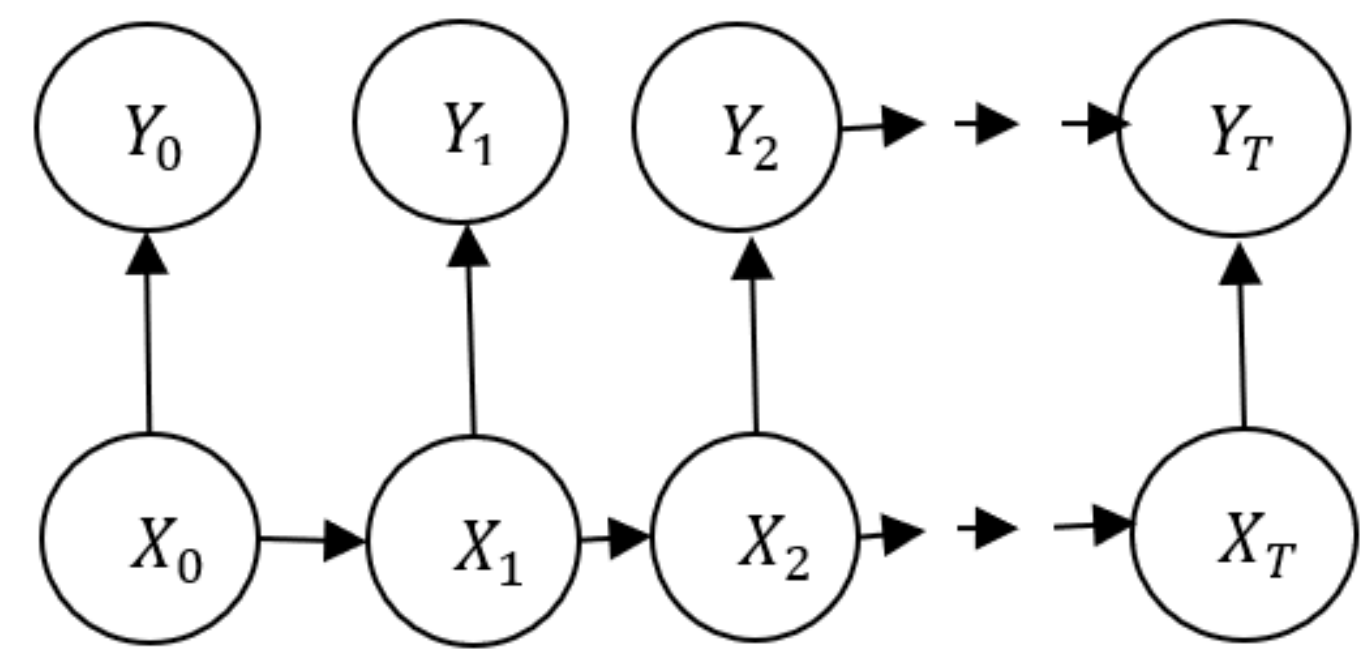

Figure 3

Principle of optimal filter evolution according to HMM [1]
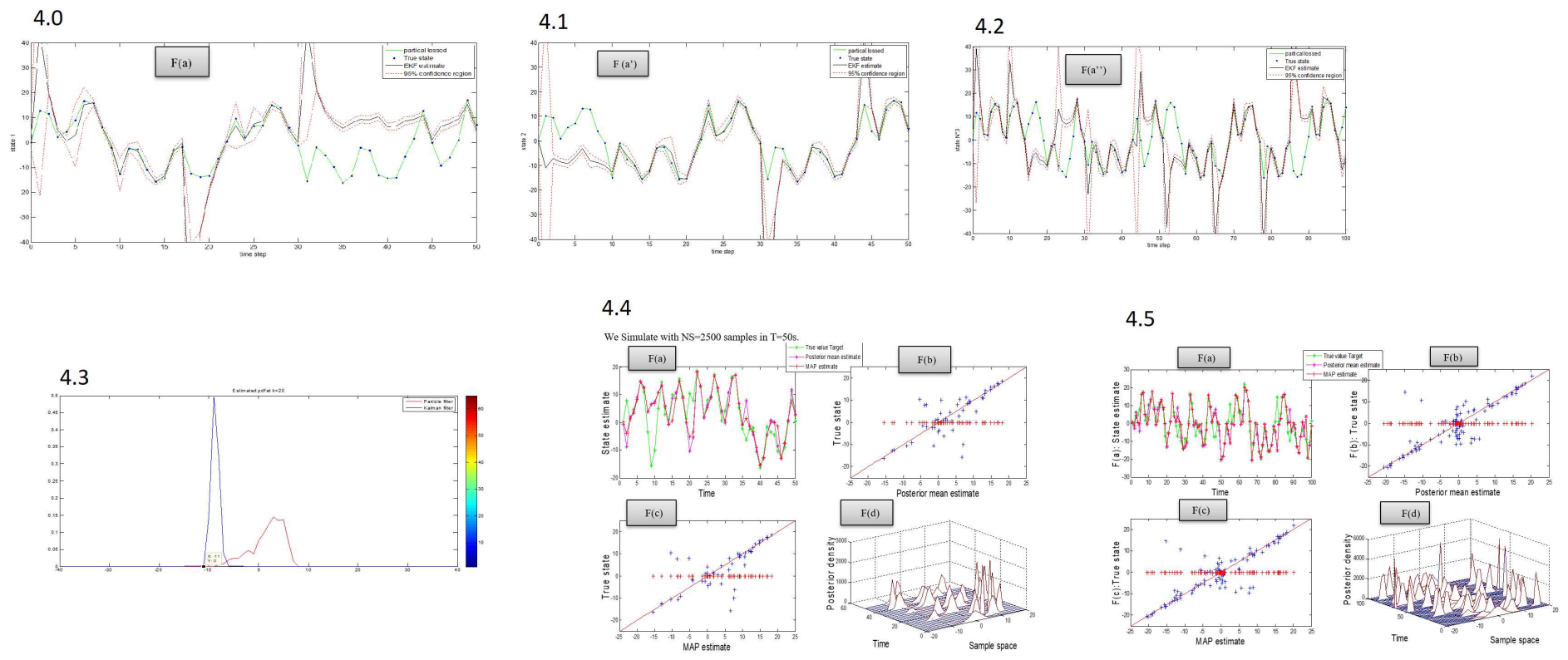

Figure 4

4.0 State estimated of the target (state $\mathrm{N}^{\circ} 1$ ) with $\mathrm{NS}=500$ and $\mathrm{T}=50 \mathrm{~s}$ respectively. 4.1 State estimated of the target $\left(F(a)\right.$ et $\left.F\left(a^{\prime}\right)\right)$ with NS=2500 and $T=50$ s respectively. 4.2 State $N^{\circ} 3$ estimated of the target with NS $=5000$ and $T=100$ s. 4.3 PF and EKF Estimation of Pdf using NS $=500$ and NS $=2500.4 .4$ State estimation of manoeuvring target using SIR algorithm with NS=2500 in T=50s. 4.5 State estimation of a manoeuvring target using SIR algorithm with NS=5000 in T=100s 